\section{Limit on the Angular Size of Cyg XR-I}

Вотн nebular and stellar objects have been identified as discrete sources of cosmic X-rays. Representative of the first is the Crab Nebula, the size of which has been measured to be $\mathrm{I}$ to 2 arc $\mathrm{min}$ in the energy range $1-6 \mathrm{keV}$ according to observations during a lunar occultation ${ }^{1}$ and with a modulation collimator ${ }^{2}$. This size is comparable with the optical size of the nebula. Representative of stellar sources is Sco $X-1$, the X-ray size of which has been shown to be less than 20 arc $\mathrm{s}$ by a measurement with a modulation collimator, also in the energy range 1-6 keV (ref. 3). The optical counterpart of Sco $X-1$ is an irregularly variable blue star. The tentative identifications of Cas $X R-1$ with the supernova remnant, Cas $A$ (ref. 5, and of several other X-ray sources with blue stars (Cyg $X-2$ (ref. 6), Cen $X R-2$ (ref. 7)) obviously suggest that these particular sources can be assigned to the nebular and the stellar classes, respectively.

The bright $\mathrm{X}$-ray source Cyg $X R-1$ presents an especially interesting problem for size measurement because it has a hard "power-law" X-ray spectrum extending to $100 \mathrm{keV}$ and beyond ${ }^{8}$ like the Crab Nebula, and is apparently highly variable ${ }^{9}$ like Sco $X-1$. Moreover, no optical counterpart of Cyg $X R-1$ has been identified. Thus a direct measurement of its $X$-ray size may be the only way to determine whether it is a variable $\mathrm{X}$-ray nebula or a stellar object emitting $\mathrm{X}$-rays with a power law spectrum extending beyond $100 \mathrm{keV}$.

This article reports an observation that places an upper limit of $1.4 \mathrm{~min}$ of arc on the angular diameter of the $\mathrm{X}$-ray source Cyg $X R-1$ in the energy range from 25 to $100 \mathrm{keV}$. This result is based on data from a balloon experiment on July 16, 1968, with an instrument com. prising a pair of X-ray telescopes with gyro-stabilized modulation collimators.

Each collimator consists of two parallel grids of $10 \mathrm{mil}$ gold wires mounted 35 inches apart in a cylindrical tube. When an X-ray source passes across the field of view along an arc perpendicular to the wires, the transmitted intensity is modulated with a period of $2 \cdot 0$ are min and an amplitude that depends on the angular diameter of the source. For a point source the modulation is nearly 100 per cent, and for a source much larger than 2 are min it is nearly zero. A slow and steady source scan is achieved by an inertial stabilization system. Two rate integrating gyroscopes ar'e used to stabilize the collimators against random motions of the balloon gondola while rotating them at a uniform rate of several are minutes per minute.

The $\mathrm{X}$-rays transmitted by the collimators are detected by two $\mathrm{NaI}$ ( $\mathrm{Tl})$ crystal scintillator detectors which are mounted behind the collimators. The total effective area of the instrument in the direction of a transmission maximum is $280 \mathrm{~cm}^{2}$. Its overall fiold of view is $10^{\circ}$ by $10^{\circ}$. full width at half maximum. The instrument is orientated in azimuth and elevation and is controlled by radio command from the ground. The X-ray counting rate in each detector is telemetered to the ground once per second and recorded.

The instrument was launched from the National Center for Atmosphoric Research (NCAR) balloon base at Palestine, Texas. Command and telemetry systems were provided by NCAR. The data gathering portion of the flight lasted for $5 \mathrm{~h} 30 \mathrm{~min}$ at an atmospheric depth of $3.74 \mathrm{~g} \mathrm{~cm}^{-2}$. A souren was observed when the telescope was pointed in the direction given by Giacconi et $a l .{ }^{10}$ for Cyg $X R-1$. The intensity of $\mathrm{Cyg} X R-1$ at the altitude of observation was found to be $0 \cdot 056 \pm 0 \cdot 007$ photons $\mathrm{cm}^{-2} \mathrm{~s}^{-1}$ in the 25 to $100 \mathrm{keV}$ energy range. Because the command equipment malfunctioned during most of the flight, only one good scan, of 8 min duration, was made of Cyg $X R-1$ by the collimators. A Fourier transformation of the data recorded during this scan revealed a strong periodicity in the $\mathrm{X}$-ray intensity at a frequency consistent with the scan rate, which was determined independently from telemetered engincering data. The probability that random fluctuations were responsible for the periodicity is $10^{-3}$; equivalent to a $3.45 \sigma$ deviation on the normal distribution curve. Possible instrumental effects which could cause an apparent modulation of X-ray intensity were investigated and found to be negligible. The same analysis was applied to data taken during the remaining $5 \mathrm{~h} 24 \mathrm{~min}$ of the flight when the instrument could be pointed toward and away from the source, but the collimators could not be stabilized. No evidence of significant periodicity was found in these other data. The Fourier coefficients computed from these other data followed the predicted probability law very closely which indicates that the statistical analysis is valid and that the probability quoted here is realistic. The observed periodicity is therefore attributed to a modulation of the Cyg $X R-1$ intensity by the collimators.

The amplitude of the periodic component indicates that the source diameter is less than 1.4 are min. The result is actually consistent with the hypothesis that Cyg $X R-1$ is a point source, but the limited observing time and relatively poor statisties introduce too much uncertainty to justify setting a smaller limit than 1.4 are min at this time.

Given sufficient observing time, this instrument is capable of measuring diameters of high energy $\mathrm{X}$-ray sources down to $0 \cdot 1$ are min. Flights are planned for both Cyg $X R-1$ and the Crab Nebula.

I thank Professor George Clark for the original concept of this work, and Professor Herbert Schnopper and other members of the staff of the MIT Center for Space Research -chiefly Messrs T. Dawson and E. Mangan---for their help. This work was supported in part by a NASA grant.

Center for Space Rescarch,

FrankLin W. HLOYD*

Massachusetts Institute of Technology.

Received March 3, 1969.

* Present address: MIT Lincoln Laboratory.

Bowyer, S., Byram, E. T., Chubb, T. A., and Friedman, H., Science, 146 912 (1964).

${ }^{2}$ Oda, M., Bradt, H., Garmire, G., Spada, G., Sreekantan, 13. V., Gursky, H., Giacconi, R., Gorenstein, P., and Waters, J. R., Astrophys. J. Lett., 148, L5 (1967).

${ }^{3}$ Gursky, H., Giacconi, R., (xorenstein, P., Waters, J. R., Oda, M., Bradt, H., Garmire, G., and Sreekantan, B. V., Ap. J., 144, 1249 (1966).

${ }^{4}$ Sandage, A., Osmer, P., Giacconi, R., Gorenstein, P., Ginsky, H., Waters, J., Bradt, H., Garmire, G., Sreekantan, B. V., Oda, M., Osawa, K., and Jugakn, J., Astrophys. J., 146, 316 (1966).

${ }^{5}$ Byram, F. T., Chubb, T. A., and Friedman, H., Science, 152, 66 (1966).

${ }^{8}$ Giacconi, R., Gorenstein, P., Gursky, H., Usher, P. D., Waters, J. R., Sandage, A., Osmer, P., and Peach, J. V., A strophys. J.' Lett., 148, I129 (1967).

' Fggen, O. J., Freeman, K. C., and Sandage, A., Astrophys. ,J. Lett., 154, L27 (1968).

${ }^{8}$ Haymes, R. C., Ellis, D. V., Fishman, G. J., Glen, S. W., and Kurfess, J. D., Astrophys. J. Lett., 151, L̇125 (1968)

${ }^{9}$ Byram, E., Chubb, T., and Friedman, H., Science, 152, 66 (1966).

${ }^{\circ}$ Giacconi, R, (Gorenstein, P., Gursky, H., and Waters, J. R., Astrophys. J. Tett., 148, L119 (1967).

\section{Interferometric Observations of the Radio Source VRO 422201}

SснмітT $^{1}$ has emphasized the outstandingly interesting properties of VRO 422201 and its associated optical counterpart $B L \mathrm{Lac}^{2}$. Further interest has been aroused recently by the supposed detection of variable circular polarization at a frequency of $2,695 \mathrm{MHz}$ (refs. 3 and 4). We have observed VRO 422201 at various times and frequencies during 1968 with the interferometer of the Owens Valley Radio Observatory, alway's in conjunction with other programmes.

The results are summarized in Table 1. The orientation of the interferometer is specified in the second and third 